\title{
Trombose venosa profunda após uma fratura supracondilar do úmero em uma criança - Uma complicação rara*
}

\section{Deep Vein Thrombosis after a Supracondylar Fracture of the Humerus in a Child - A Rare Complication}

\author{
Ana Moreira Ferrão ${ }^{10}$ Pedro Fernandes ${ }^{2}$ Patrícia Wircker ${ }^{3}$ Joana Catarino ${ }^{4}$ Joana Arcângelo ${ }^{5}$ \\ Joana Ovídio ${ }^{5}$
}

1 Serviço de Ortopedia e Traumatologia, Hospital de Curry Cabral, Centro Hospitalar Universitário de Lisboa Central, Portugal

Endereço para correspondência Ana Moreira Ferrão, Serviço de Ortopedia e Traumatologia do Centro Hospitalar Universitário de

2 Serviço de Ortopedia e Traumatologia, Centro Hospitalar de Lisboa Ocidental, Portugal

${ }^{3}$ Serviço de Ortopedia e Traumatologia, Hospital de Cascais, Portugal Lisboa Central, Rua da Beneficência, 8, 1050-099, Lisboa, Portugal (e-mail: anamoreiraferrao@gmail.com).

${ }^{4}$ Serviço de Cirurgia Vascular, Hospital de Santa Marta, Centro

Hospitalar Universitário de Lisboa Central, Portugal

${ }^{5}$ Serviço de Ortopedia Infantil, Hospital de Dona Estefânia, Centro

Hospitalar Universitário de Lisboa Central, Portugal

Rev Bras Ortop

\section{Resumo \\ Palavras-chave \\ - trombose venosa profunda \\ - fraturas do úmero \\ - trombose \\ - tromboembolia venosa \\ - criança}

Trombose venosa profunda nas extremidades superiores é incomum, especialmente na população pediátrica e no ambiente do trauma. O diagnóstico é desafiador, devido a sua raridade, exigindo alto grau de suspeita.

Descrevemos um caso raro de trombose venosa úmera após uma fratura supracondilar deslocada do úmero em uma menina de 7 anos. Os fatores de risco para tromboembolismo e sequelas também são discutidos.

A detecção e o tratamento precoces são obrigatórios para evitar desfechos ruins, como tromboembolismo fatal.
Trabalho desenvolvido no Serviço de Ortopedia e Traumatologia do
Centro Hospitalar Universitário de Lisboa Central, Lisboa, Portugal.

recebido

02 de Junho de 2020

aceito

16 de Setembro de 2020
DOI https://doi.org/

10.1055/s-0040-1722580.

ISSN 0102-3616. (c) 2021. Sociedade Brasileira de Ortopedia e Traumatologia. All rights reserved.

This is an open access article published by Thieme under the terms of the Creative Commons Attribution-NonDerivative-NonCommercial-License, permitting copying and reproduction so long as the original work is given appropriate credit. Contents may not be used for commercial purposes, or adapted, remixed, transformed or built upon. (https://creativecommons.org/ licenses/by-nc-nd/4.0/)

Thieme Revinter Publicações Ltda., Rua do Matoso 170, Rio de Janeiro, RJ, CEP 20270-135, Brazil 


Abstract
Keywords
- deep vein thrombosis
- humeral fractures
- thrombosis
- venous
thromboembolism
- child

Deep vein thrombosis in the upper extremities is uncommon, especially in the pediatric population and in the trauma setting. The diagnosis is challenging, due to its rarity, requiring a high degree of suspicion.

We describe a rare case of humeral vein thrombosis after a displaced supracondylar fracture of the humerus in a 7-year-old girl. The risk factors for thromboembolism and sequelae are also discussed.

The early detection and treatment are mandatory to prevent poor outcomes, such as fatal thromboembolism.

\section{Introdução}

As fraturas supracondilares umerais representam um terço de todas as fraturas pediátricas de membros em crianças com menos de 7 anos de idade, e são uma causa de morbidade significativa na população pediátrica., ${ }^{1,2}$

As complicações mais encontradas neste cenário incluem lesões neurovasculares, síndrome compartimental, má consolidação e comprometimento funcional. ${ }^{1,2}$

Lesões nervosas e vasculares podem ser iatrogênicas ou podem ocorrer após um evento traumático. 0 comprometimento vascular pode ser causado por ruptura do vaso, torção, compressão, espasmo ou lesão intimal, e a incidência aumenta com o grau de luxação da fratura. ${ }^{3}$

A incidência geral de complicações vasculares associadas a fraturas supracondilares varia de $3,2 \%$ a $14,3 \%$, mas pode ser de até $20 \%$ nas fraturas de tipo III de Gartland. ${ }^{3,4}$ Na inspeção, equimose na fossa antecubital com pele e braquial são sinais de alerta de dano neurovascular. ${ }^{4}$ Embora a incidência e o manejo de lesões arteriais em fraturas supracondilares sejam bem estabelecidos na literatura, pouco tem sido dito sobre o comprometimento venoso.

O tromboembolismo venoso (TEV) em crianças é raro, e varia de $0,01 \%$ a $0,2 \%$ das internações pediátricas; taxas mais elevadas são observadas em grupos específicos, incluindo aqueles com cateteres venosos centrais (CVCs), malignidades, obesidade, e os que foram submetidos a cirurgias graves. ${ }^{5,6}$

Em pacientes com trauma pediátrico, o risco de TEVé incerto. A gravidade da lesão, o aumento da idade, a anemia, a fixação externa, a obesidade, o tempo de internação hospitalar, as complicações pós-operatórias, a lesão medular e a presença de um CVC são fatores de risco bem conhecidos nesta população. ${ }^{6-8}$ Alguns locais anatômicos também são mais propensos a eventos trombóticos, ou seja, trauma de cabeça, coluna ou vasos principais, e fraturas pélvicas e dos membros inferiores. ${ }^{8}$

Relatamos um caso de trombose venosa umeral após uma fratura supracondilar em uma criança, dada a sua raridade e difícil diagnóstico, considerando suas possíveis complicações.

\section{Relato de Caso}

Uma menina de 7 anos foi transferida para o pronto-socorro após uma queda com o braço estendido que resultou em uma fratura supracondilar do úmero esquerdo de tipo III de Gartland (-Fig. 1). Ela havia sido imobilizada anteriormente com uma tala posterior em um hospital externo.
Na apresentação, ela reclamou de dor, e tinha o antebraço frio e avermelhado, com saturação periférica de oxigênio de 89\% a 90\%, e pulsos radial e ulnar palpáveis fracos (-Fig. 2).

Após a remoção da tala, a dor diminuiu, ela tinha pulso radial fraco, recarga capilar normal, e a oximetria da mão chegou a $98 \%$. Em regime de urgência, a paciente foi submetida a uma manipulação suave com redução fechada e fixação com 2 fios de Kirschner laterais divergentes e imobilização em tala posterior a $120^{\circ}$ de flexão ( - Fig. 3). Seis horas após o operatório, a criança tinha uma extremidade superior livre de dor e quente, uma saturação de oxigênio periférico de $100 \%$, e pulsos distais palpáveis.

Quarenta e oito horas após a cirurgia, houve dor persistente e edema sensível progressivo, com bolhas de pele

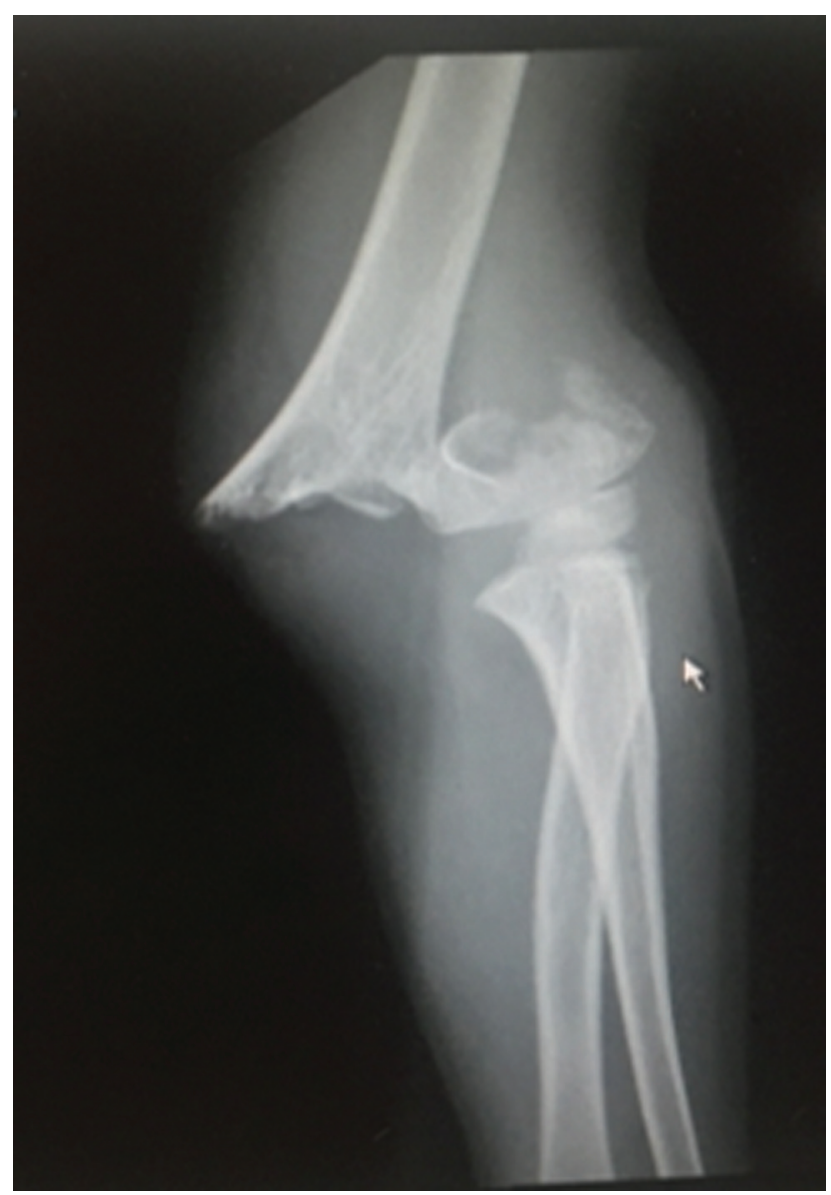

Fig. 1 Imagem radiográfica da fratura supracondilar do úmero (tipo III de Gartland). 


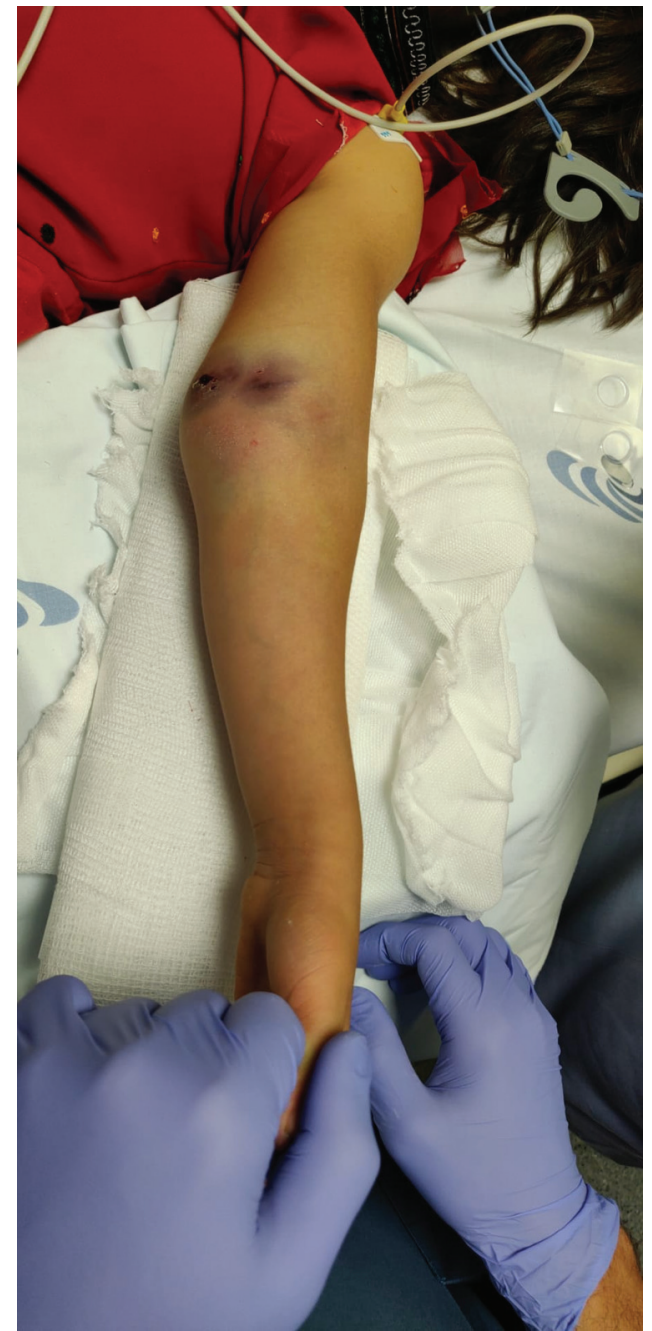

Fig. 2 Equimose na fossa antecubital.

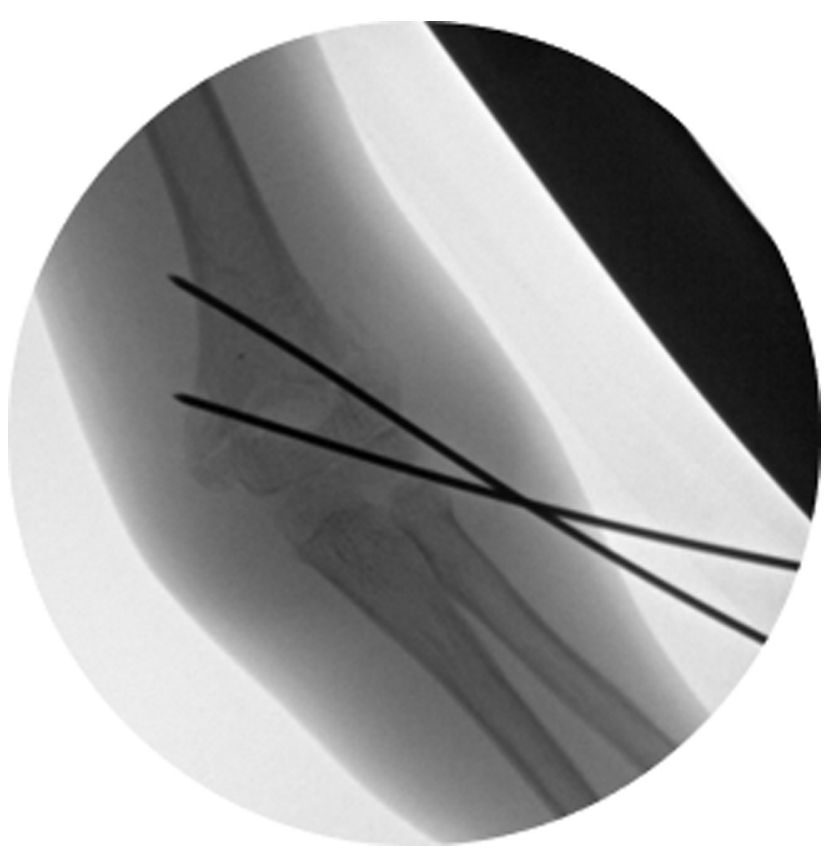

Fig. 3 Redução e fixação fechada com dois fios de Kirschner percutâneos.

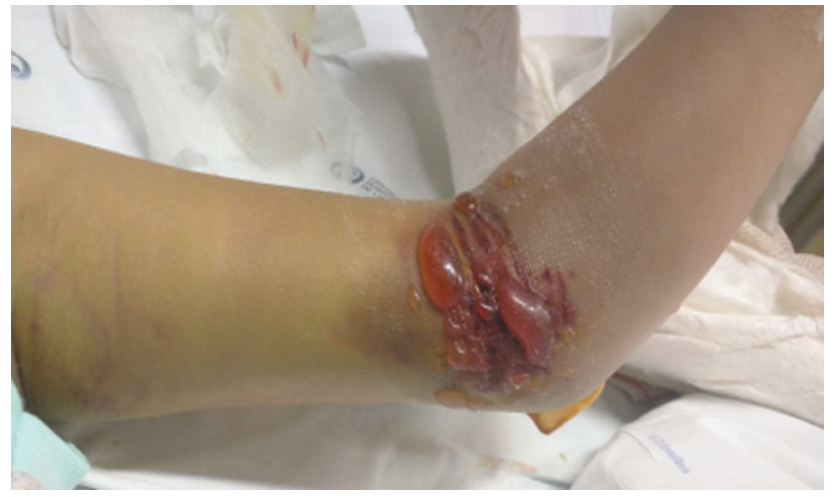

Fig. 4 Edema e bolhas na pele 48 horas após a cirurgia.

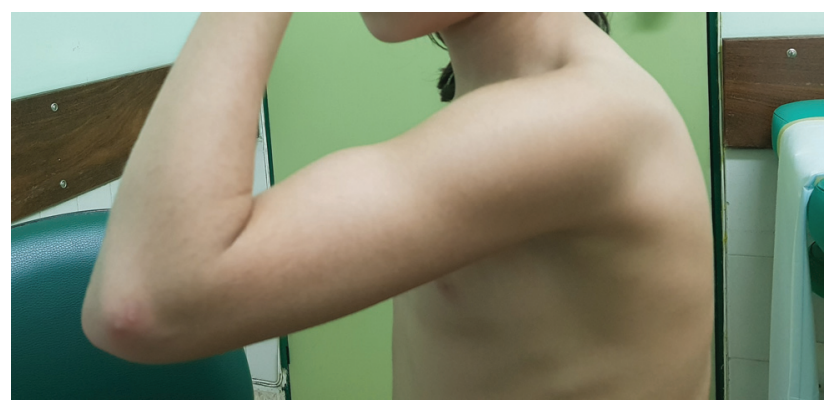

Fig. 5 Faixa de movimento em três meses de acompanhamento.

(-Fig.4). Os pulsos periféricos estavam normais, e não houve exacerbação da dor com mobilização passiva dos dedos. Após a exclusão da síndrome compartimental, foi solicitada uma ultrassonografia duplex venosa, que revelou uma trombose venosa profunda (TVP) da veia umeral.

O tratamento com heparina subcutânea de baixo peso molecular de $20 \mathrm{mg}$ foi iniciado e mantido por 3 meses. Uma semana após a cirurgia, a criança estava livre de dor, com melhora do edema, e recebeu alta hospitalar. Aos 3 meses de seguimento, a fratura foi curada, e o cotovelo teve amplitude total de movimento do cotovelo $\left(0^{\circ}\right.$ a $\left.120^{\circ}\right)$, sem complicações adicionais de pele ou vasculares (-Figs. 5 e $\mathbf{6}$ ). Não houve eventos embólicos. Os estudos realizados foram negativos para trombofilia.

\section{Discussão}

O manejo de fraturas supracondilares do úmero pode ser exigente. As complicações precoces incluem danos às estruturas neurovasculares ou musculares e síndrome compartimental. ${ }^{9}$ Lesões neurológicas, mais frequentemente na forma de neuropraxia, são comuns ( $\sim 20 \%$ ), e afetamo principalmente o nervo mediano e seu ramo interósseo anterior., ${ }^{3,9}$ As lesões mais desastrosas são vasculares, pois, quando não tratadas, podem levar a déficits neurológicos, rigidez muscular, ou contraturas isquêmicas de Volkmann. ${ }^{9}$ Complicações relacionadas ao hardware e sequelas de má consolidação geralmente são eventos posteriores. $^{9}$

De acordo com a literatura, os pacientes com trauma pediátrico têm prevalência de TEV de 0,3 a 0,8 para cada mil altas de trauma. ${ }^{7}$ Além disso, cirurgias emergentes ou 


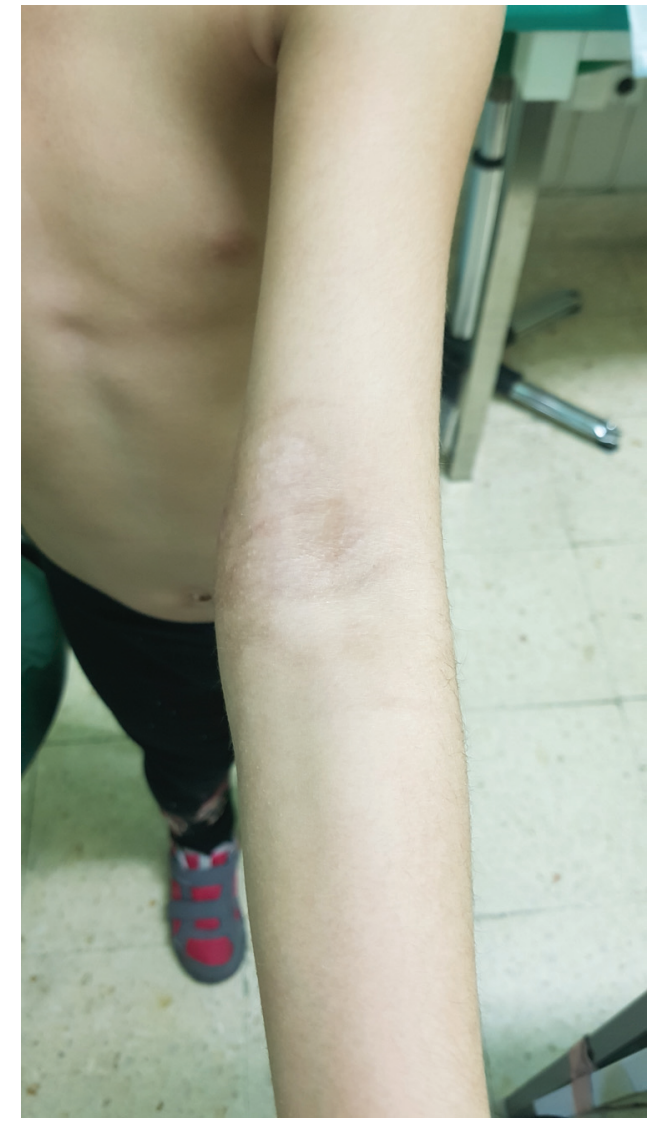

Fig. 6 Pele intacta e extensão completa em três meses de acompanhamento.

urgentes são mais propensas a resultar em TEV do que procedimentos eletivos. $^{6}$

Para que o diagnóstico seja feito, é necessária suspeita clínica, e um exame vascular detalhado é crucial, com avaliação de pulsos, temperatura do membro, retorno capilar e oximetria do pulso. ${ }^{3} \mathrm{O}$ uso de ultrassom intraoperatório ou pós-operatório também é útil para avaliar a patência dos vasos. ${ }^{4}$ Em alguns casos, a forma de onda de oximetria de pulso pode ser usada para determinar a necessidade de exploração vascular. ${ }^{10}$

Sequelas de tromboembolismo venoso, ou seja, síndrome pós-trombolítica, risco de morte e recorrência não foram totalmente investigados em crianças. ${ }^{5}$ A morbidade da TVP depende da localização e gravidade da lesão, induzindo principalmente dor e inchaço quando nas extremidades. ${ }^{5}$

O TEV em crianças está associado ao aumento da mortalidade, particularmente nos pacientes mais jovens, variando entre $1 \%$ e $8 \%{ }^{5,6}$ Guzman et al. ${ }^{7}$ procuraram associação entre TEV e mortalidade em crianças, e concluíram que pacientes com fraturas de membros superiores e TEV apresentaram a maior taxa global de mortalidade (6,4\%). Acredita-se que esse aumento da mortalidade seja devido a um atraso no diagnóstico e tratamento (devido a um menor índice de suspeita), e/ou pela proximidade anatômica das veias da extremidade superior às câmaras do coração do lado direito. ${ }^{7}$

O motivo para identificar os grupos de risco para TEV entre as crianças é prevenir a morte secundária a uma embolia pulmonar. ${ }^{7}$ Atualmente, não há consenso para tromboprofilaxia no campo pediátrico, nem estratificação de risco para TEV., 5,6

Dada a rara incidência de TEV nesta faixa etária, os atrasos no diagnóstico são frequentes. No nosso caso, o edema, a coloração da pele e as bolhas foram cruciais para levantar suspeitas. Até agora, há muitos estudos focados em complicações arteriais e nervosas, mas nenhum sobre TEV neste cenário.

O objetivo deste relato é chamar a atenção para uma rara complicação vascular de uma fratura supracondilar umeral. Pelo que sabemos, este é o primeiro relato desta complicação.

Todos os cirurgiões ortopédicos devem estar cientes da existência de TVP de extremidade superior para fazer um diagnóstico precoce.

Recomendamos, portanto, um exame neurovascular detalhado antes e depois da cirurgia, e, se a suspeita clínica for alta, uma avaliação com ultrassom com Doppler.

\section{Declaração de Ética}

Este estudo foi realizado em conformidade com a Declaração da Associação Médica Mundial de Helsinque sobre Princípios Éticos para Pesquisa Médica Envolvendo Sujeitos Humanos.

\section{Conflito de Interesses}

Os autores declaram não haver conflito de interesses.

\section{Referências}

1 Claireaux H, Goodall R, Hill J, et al. Multicentre collaborative cohort study of the use of Kirschner wires for the management of supracondylar fractures in children. Chin J Traumatol 2019;22(05):249-254

2 Saarinen AJ, Helenius I. Paediatric supracondylar humeral fractures: the effect of the surgical specialty on the outcomes. J Child Orthop 2019;13(01):40-46

3 Leiblein M, Lustenberger T, Schulz A-K, Schmitz-Rixen T, Marzi I. Neurovascular complications after supracondylar humerus fractures in children. Trauma Case Rep 2017;8:16-19

4 Louahem D, Cottalorda J. Acute ischemia and pink pulseless hand in 68 of 404 gartland type III supracondylar humeral fractures in children: Urgent management and therapeutic consensus. Injury 2016;47(04):848-852

5 Mahajerin A, Croteau SE. Epidemiology and Risk Assessment of Pediatric Venous Thromboembolism. Front Pediatr 2017;5:68

6 Baker D, Sherrod B, McGwin G Jr, Ponce B, Gilbert S. Complications and 30-day outcomes associated with venous thromboembolism in the pediatric orthopaedic surgical population. J Am Acad Orthop Surg 2016;24(03):196-206

7 Guzman D, Sabharwal S, Zhao C, Sabharwal S. Venous thromboembolism among pediatric orthopedic trauma patients: a database analysis. J Pediatr Orthop B 2018;27(02):93-98

8 O'Brien SH, Candrilli SD. In the absence of a central venous catheter, risk of venous thromboembolism is low in critically injured children, adolescents, and young adults: evidence from the National Trauma Data Bank. Pediatr Crit Care Med 2011;12(03):251-256

9 Tomaszewski R, Wozowicz A, Wysocka-Wojakiewicz P. Analysis of Early Neurovascular Complications of Pediatric Supracondylar Humerus Fractures: A Long-Term Observation. BioMed Res Int 2017;2017:2803790

10 Soh RCC, Tawng DK, Mahadev A. Pulse oximetry for the diagnosis and prediction for surgical exploration in the pulseless perfused hand as a result of supracondylar fractures of the distal humerus. Clin Orthop Surg 2013;5(01):74-81 